\title{
Pancreatitis recurrente en pediatría: serie de casos
} Recurrent pancreatitis in children: Case series

\author{
Dra. Ma. Alejandra Mortarini ${ }^{a}$ Dra. Johana Hincapie Butto ${ }^{a}$, Dra. Sandra Basso y Dra. Ana Rocca ${ }^{a}$
}

\begin{abstract}
RESUMEN
La pancreatitis recurrente (PR) ocurre en el 15-36\% de las pancreatitis agudas, en la edad pediátrica. Se realizó un estudio descriptivo y transversal, para determinar la etiología y la evolución en menores de 18 años con diagnóstico de PR entre 2008-2016 en el Hospital Garrahan. Se incluyeron 10 pacientes, el $90 \%$ de sexo femenino. La mediana de edad fue de 11 años. La mediana del número de episodios fue de nueve. La ecografía abdominal y/o la colangioresonancia iniciales mostraron hallazgos patológicos en cuatro pacientes. De los seis pacientes con estudios iniciales normales, tres desarrollaron signos de pancreatitis crónica (PC), uno con estenosis del Wirsung sugestiva de pancreatitis autoinmune. El $40 \%$ de los pacientes tuvo diagnóstico etiológico. Enel seguimiento, tres pacientes con pancreatitis idiopática desarrollaron PC. Los factores genéticos podrían jugar un papel en los casos considerados idiopáticos. Palabras clave: pancreatitis, pancreatitis crónica, pancreatitis aguda necrosante.
\end{abstract}

\begin{abstract}
Recurrent pancreatitis (RP) occurs in children between 15$35 \%$ of the cases. To determine the etiology and outcome of RP in children a descriptive, cross-sectional cohort study was conducted in children under 18 years of age with RP seen at Hospital Garrahan between 2008-2016. Of 10 patients with RP, $90 \%$ were girls. Median age of the diagnoses of RP was 11. Median number of episodes was 9. Initial abdominal ultrasonography and/or magnetic resonance cholangiopancreatography were abnormal in four patients. Of six patients with normal studies at onset, three developed signs of chronic pancreatitis (CP) and one stenosis of the duct of Wirsung suggestive of autoimmune pancreatitis. In $40 \%$ of our patients, the etiology was determined. On follow-up, three patients with idiopathic pancreatitis developed CP. Genetic factors may play a role in patients considered to have idiopathic pancreatitis.
\end{abstract}

Key words: Pancreatitis, chronic pancreatitis, acute necrotizing pancreatitis.

a. Servicio de Gastroenterología, Hospital de Pediatría SAMIC "Prof. Dr. Juan P. Garrahan", Ciudad Autónoma de Buenos Aires, Argentina.

b. Servicio de Páncreas, Hospital Bonorino Udaondo, Ciudad Autónoma de Buenos Aires, Argentina.

Correspondencia:

Dra. Ma. Alejandra Mortarini: alimortarini@gmail.com

Financiamiento: Ninguno.

Conflicto de intereses: Ninguno que declarar.

Recibido: 8-11-2019

Aceptado: 20-1-2021 http: / / dx.doi.org/10.5546/ aap.2021.e322

Cómo citar: Mortarini MA, Hincapie Butto J, Basso S, Rocca A Pancreatitis recurrente en pediatría: serie de casos. Arch Argent Pediatr 2021;119(4):e322-e325.

\section{INTRODUCCIÓN}

La pancreatitis recurrente (PR) ocurre en un 15 a $36 \%$ de los niños con pancreatitis aguda. ${ }^{1}$ Según el grupo INSPPIRE (International Study Group of Pediatric Pancreatitis: In search for a cure) se define como dos o más episodios distintos de pancreatitis aguda (PA) con resolución completa del dolor (con por lo menos un mes de intervalo) o normalización completa de enzimas pancreáticas antes del diagnóstico del próximo episodio junto con resolución del dolor, independientemente del tiempo transcurrido entre episodios de PA. ${ }^{2}$ Las causas pueden ser de origen obstructivo o no obstructivo. Dentro de las primeras se describen el quiste de colédoco, páncreas divisum, divertículo duodenal, duplicación duodenal, infección parasitaria y anomalías de la unión pancreatobiliar. Las causas no obstructivas incluyen factores hereditarios, autoinmunes, fibrosis quística (FQ), hiperlipidemia, trauma, medicamentos e hipercalcemia. Si a pesar de la búsqueda exhaustiva no se identifica ninguna causa, se hablará de pancreatitis recurrente idiopática. $^{3}$

En la actualidad, la genética y medicina molecular juegan un papel importante en la búsqueda etiológica de las PR. La importancia de determinar la etiología de la PR radica en evitar el dolor recurrente y, sobre todo, la insuficiencia pancreática irreversible con todas las complicaciones que conlleva.

\section{POBLACIÓN Y MÉTODOS}

Se realizó un estudio descriptivo de cohorte transversal, retrospectivo desde la recolección de datos, en pacientes de 0-18 años del Hospital Garrahan con diagnóstico de PR en el período 2008-2016.

Criterios de inclusión: pacientes menores de 18 años con PR sin diagnóstico etiológico al inicio de la evaluación. 
Criterios de exclusión: pacientes con malformación de la vía biliar conocida previamente, pancreatitis postraumáticas o infecciosas, diagnóstico previo de fibrosis quística y pancreatitis asociada a enfermedades crónicas graves.

El estudio fue aprobado por el Departamento de Investigación del Hospital.

\section{RESULTADOS}

En el período estudiado ingresaron al centro 10 pacientes con diagnóstico de PR $(90 \%$ de sexo femenino). La mediana de edad fue de 11 años, y la mediana de edad del primer episodio fue de 5,5 años (rango 2 a 15). La mediana en relación con el número de episodios fue de 9 (rango 3 a 12).

Solo un paciente tuvo un puntaje $Z+2$. Un paciente desarrolló PA grave.

En seis de los 10 pacientes encontramos valores de amilasa $>1000 \mathrm{U} / \mathrm{L}$ durante el episodio agudo de pancreatitis. Todos los pacientes presentaron anticuerpos negativos para enfermedad celíaca. La determinación de autoanticuerpos automúsculo liso (ASMA) con especificidad actina fue positiva en 7 de 8 pacientes. La IgG4 fue débilmente positiva en 2 de 8 pacientes. Uno perdió seguimiento y la otra paciente, con dosaje de IgG4 levemente superior al valor de corte, no presentaba otros elementos sugestivos de pancreatitis autoinmune (PAI), por lo que no se asumió como tal.

En siete pacientes se realizó el test del sudor (sistema de recolección Macroduct ${ }^{\circledR}$ ) y búsqueda de mutaciones para FQ DF508 y 31 mutaciones más (Kit Cystic Fibrosis GenotypingAssay Abbott ${ }^{\circledR}$ ). Un paciente presentó test del sudor patológico en dos oportunidades y estudio molecular con mutación DF508 heterocigota, con lo que se confirmó el diagnóstico de FQ. Al momento de la evaluación, el paciente presentaba suficiencia pancreática.

Se evaluó la función pancreática exocrina en todos los pacientes, con elastasa y prueba de Van de Kamer en materia fecal. Solo un paciente presentó valores de elastasa $<200 \mu \mathrm{g} / \mathrm{g}$ y alteración en la prueba el Van de Kamer, asumida como insuficiencia pancreática exocrina.

En los estudios por imágenes (ecografía abdominal y/o colangiorresonancia) se encontraron hallazgos iniciales patológicos en 4 pacientes. Se observó: 1) malformación de la vía biliar Todani de tipo IVa, 2) páncreas divisum, 3) barro biliar y 4) páncreas aumentado de tamaño. De los 6 pacientes con estudios iniciales normales, 3 desarrollaron signos sugestivos de PC (dilatación del conducto de Wirsung, cambios en el tamaño y contorno irregular del páncreas) en su evolución, uno de ellos con estenosis del Wirsung sugestiva de pancreatitis autoinmune. En ningún caso se observaron calcificaciones.

Dos pacientes presentaron antecedentes de autoinmunidad familiar, como hipotiroidismo y tiroiditis de Hashimoto en familiares de primer grado. Ninguno de los pacientes presentó antecedentes familiares de pancreatitis.

El diagnóstico etiológico se pudo establecer en 4 de los 10 pacientes estudiados, dos de ellos con malformación de la vía biliar de resolución quirúrgica, en un caso se confirmó FQ y otro fue asumido como PAI. Dos de nuestros pacientes perdieron seguimiento durante su evolución.

\section{DISCUSIÓN}

La PR es una enfermedad poco frecuente en pediatría, pero de magnitud creciente en los últimos años. Ocurre en 15-35 \% de los niños con PA. ${ }^{1}$

Realizamos una búsqueda retrospectiva de los pacientes ingresados con PA, se encontraron diez pacientes con PR a lo largo de ocho años. Se observó una amplia predominancia del sexo femenino $(90 \%)$, que no coincide con lo publicado. $^{4-5}$

La edad de presentación del primer episodio (mediana 5,5 años) fue más baja que la encontrada en la bibliografía. ${ }^{6}$

Dentro de las etiologías probables relacionadas con la PR, se considera que la alteración primaria del metabolismo de los lípidos como la hipertrigliceridemia familiar, la hiperlipidemia de tipos IV y V o secundaria como obesidad y/o diabetes, pueden causar alteraciones pancreáticas. Su papel en la etiología de la PA es motivo de discusión. ${ }^{7}$ En nuestra serie, solo un paciente presentó índice de masa corporal elevado y ninguno tuvo alteración del lipidograma. A pesar que en nuestro estudio no encontramos ningún paciente con enfermedad celiaca, esta debe tenerse en cuenta en todo paciente con PR, dado que se trata de una entidad frecuente en nuestra población que, asociada a estados de desnutrición, estenosis papilar y por mecanismos inmunopatogenéticos ya conocidos, contribuyen al desarrollo de PA y PR e, incluso, puede llegar a enmascarar una PAI. ${ }^{7,8}$

La PAI es una causa infrecuente de PR, sobre todo en pediatría. ${ }^{9}$ Según el consenso 
internacional de 2011, se describen dos tipos de PAI con diferentes formas clínicas, histopatológicas, asociaciones y evolución clínica. ${ }^{10}$ La mayoría de los pacientes pediátricos reportados corresponden al tipo 2, que cursa con dosaje normal de IgG4 y es más frecuente en jóvenes..$^{10}$ Solicitamos dosaje de IgG4 a los pacientes con PR sin otro diagnóstico etiológico. En 2 pacientes, la determinación fue débilmente positiva sin otros hallazgos de PAI, por lo que se desestimó. Sin embargo, un paciente que presentaba dosaje de IgG4 normal y suficiencia pancreática, finalmente fue asumido como de etiología autoinmune por hallazgos de CRM, antecedentes de autoinmunidad personal y familiar; este caso es motivo de otra publicación. ${ }^{11}$

Es conocida la asociación entre PR y FQ, se han documentado mutaciones leves del gen CFTR en pancreatitis crónicas idiopáticas. Las mutaciones graves (clases I a VI) se observan en el $92 \%$ de los casos y suelen asociarse a insuficiencia pancreática exocrina; las formas leves (clases IV-V) suelen cursar con suficiencia pancreática. ${ }^{12}$ En la mayor cohorte pediátrica de PR y PC, la frecuencia de las mutaciones del gen CFTR fue del $34 \%$ y el $23 \%$, respectivamente. ${ }^{13}$

Pant y col., ${ }^{14}$ evaluaron a 44 pacientes con PR y sugieren la necesidad de realizar un estudio detallado que incluya resonancia magnética o tomografía del páncreas para evaluar malformaciones anatómicas y mutaciones genéticas que predisponen a PR.

En cuanto a los factores genéticos, las mutaciones más frecuentes involucran los siguientes genes: PRSS1, SPINK1, CTR, el gen CFTR y, más recientemente, CPA1,CASR, $S B D S, C L D N 2$ asociados a un mayor riesgo que la población general de desarrollar PR. ${ }^{15}$ Una pérdida de la función (como ocurre en SPINK1, CTRC o CFTR) o una ganancia (PRSS1) que puede conducir a una activación prematura de tripsina o a la disminución de la capacidad inhibitoria. La mutación del PRSS1 está presente en el $80 \%$ de los casos con PC de herencia autosómica dominante. Su mayor implicancia radica en la fuerte asociación con el desarrollo de cáncer de páncreas. Se observa que los pacientes con SPINK1 tienen mayor riesgo de desarrollar PC. ${ }^{15}$ En el futuro, sería deseable determinar la presencia de esta mutación a fin de realizar un seguimiento cercano de los pacientes que son portadores.

En nuestra casuística de PR, se llegó al diagnóstico etiológico en el $40 \%$ de los casos.
Las condiciones halladas fueron malformaciones de la vía biliar, fibrosis quística y pancreatitis autoinmune. Como limitante de nuestro estudio, se evaluaron solo mutaciones del gen CFTR.

En el seguimiento, 3 pacientes con pancreatitis idiopática presentaron estudios por imágenes con hallazgos compatibles con PC. Esto coincide con los hallazgos de Schwarzenberg y col., ${ }^{5}$, que consistieron en anomalías ductales y atrofia pancreática sin calcificaciones.

Por ser un estudio retrospectivo no todos los pacientes se abordaron con el mismo protocolo. Creemos que sería apropiado elaborar un protocolo estandarizado para el abordaje y seguimiento para disminuir las recurrencias de PA, minimizar el deterioro de la función pancreática, mejorar la calidad de vida y detectar de manera temprana las complicaciones como consecuencia de un proceso inflamatorio recurrente o crónico.

La PR es infrecuente en pediatría, aunque su incidencia se encuentra en aumento. No siempre es posible hallar su causa, se recomienda la búsqueda exhaustiva para evitar la progresión a daños crónicos del páncreas y su funcionalidad.

\section{REFERENCIAS}

1. Sathiyasekaran M, Biradar V, Ramaswamy G, Srinivas S, et al. Pancreatitis in Children. Indian J Pediatr. 2016; 83(1213):1459-72.

2. Morinville VD, Husain SZ, Bai H, Barth B, et al. Definitions of pediatric pancreatitis and survey of present clinical practices. J Pediatr Gastroenterol Nutr. 2012; 5(3):261-5.

3. Hidalgo Arzola OA. Pancreatitis aguda recurrente en niños. VITAE. 2007;(31). Disponible en: http: / / www.bioline.org. $\mathrm{br} / \mathrm{pdf}$ ?va07014

4. Uc A, Yen E, Wilschanski M, Werlin S, et al. Pediatric Acute Recurrent and Chronic Pancreatitis: Report From INSPPIRE Consortium. Gastroenterology. 2014; 146 (5 Supp 1):S4.

5. Schwarzenberg SJ, Bellin M, Husain SZ, Ahuja M, et al. Pediatric chronic pancreatitis is associated with genetic risk factors and substantial disease burden. J Pediatr. 2015; 166(4):890-6.e1.

6. Lucidi V, Alghisi F, Dall'Oglio L, D'Apice M, et al. The etiology of acute recurrent pancreatitis in children: a challenge for pediatricians. Pancreas. 2011; 40(4):517-21.

7. Wejnarska K, Kolodziejczyk E, Wertheim-Tysarowska K, Dadalski M, et al The etiology and clinical course of chronic pancreatitis in children with the early-onset of the disease. J Pediatr Gastroenterol Nutr. 2016; 63(6):665-70.

8. Patel BJ, Cantor M, Retrosi G, Gheorghe R, et al. Autoimmune Pancreatitis Masqueradingas Celiac Disease. Pancreas. 2019; 48(6):e53-4.

9. Pagliari D, Cianci R, Rigante D. The challenge of autoimmune pancreatitis: a portrayal from the pediatric perspective. Pancreas. 2019; 48(5):605-12.

10. Shimosegawa T, Chari ST, Frulloni L, Kamisawa T, et al. International consensus diagnostic criteria for autoimmune pancreatitis: guidelines of the International Association of Pancreatology. Pancreas. 2011; 40(3):352-8. 
11. Mortarini A, Hincapie J, Rocca A. Pancreatitis autoinmune en pediatría. Caso clínico. Arch Argent Pediatr. [en prensa].

12. Baldwin C, Zerofsky M, Sathe M, Troendle D, et al. Acute recurrent and chronic pancreatitis as initial manifestations of cystic fibrosis and cystic fibrosis transmembrane conductance regulator-related disorders. Pancreas. 2019; 48(7):888-93.

13. Kumar S, Ooi C, Werlin S, Abu-El-Haija M, et al. Risk factors associated with pediatric acute recurrent and chronic pancreatitis: lessons from INSPPIRE. JAMA Pediatr. 2016; 170(6):562-9.
14. Pant C, Sferra TJ, Lee BR, Cocjin J, et al. Acute recurrent pancreatitis in children: a study from the Pediatric Health Information System. J Pediatr Gastroenterol Nutr. 2016; 62(3);450-2.

15. Abu-El-Haija M, Valencia CA, Hornung L, Youssef N, et al. Genetic variants in acute, acute recurrent and chronic pancreatitis affect the progression of disease in children. Pancreatology. 2019; 19(4):535-40. 